\title{
RACIONALIDAD Y LENGUAJE
}

\section{Carmen Gaona Pisonero}

Universidad Católica San Antonio de Murcia (UCAM), España.

\section{Resumen:}

Este texto integra una visión lineal de los giros lingüísticos estableciendo entre ellos un nexo de conexión: el lugar que ocupa o el papel que desempeña la razón en ellos, pese a la aparente necesaria ausencia de ésta en el lenguaje, desde una perspectiva wittgensteniana. Tomamos por tanto como eje temático lenguaje simbólico versus razón, para entender cómo actúa o cómo está en aparente ausencia la razón en el giro lingüístico analítico, en el giro pragmático, en el giro hermenéutico y en el giro pragmático- trascendental. Los frutos de dicha reflexión repercutirán en nuestra comprensión como seres comunicativos y por ende como seres culturales, desde una perspectiva comunicativa de la cultura.

Palabras clave: Lenguaje - Giro lingüístico - Semiótica - Teoría de la Información - Comunicación

\section{INTRODUCCIÓN}

En ocasiones pensamos que nuestro uso del lenguaje es algo lineal, que el querer nombrar con palabras todo lo que existe en el mundo (capacidad de referencia) y difundirlos por canales cada vez más tecnológicos y veloces, no es más que un mero deseo inteligente. La interconexión casi infinita a nivel espacio y tiempo existe y configura parte de nuestras prácticas comunicativas, pero en modo alguno la interconexión viene regulada tan solo por unas redes de área local o unos recursos informáticos conectados. No obstante, el punto de partida sigue siendo el signo y uno de sus motores de arranque la competencia comunicativa, teniendo presente que no usamos palabras reguladas por una competencia comunicativa, sino acontecimientos lingüísticos, eventos históricos sistematizados por la competencia comunicativa. Como dice J. L. Austin, dejando ver la presencia que ocupa el lenguaje vivo en un contexto sociocultural concreto, "los enunciados se hacen; las palabras o las oraciones se usan (...) un enunciado se hace, y el hacerlo es un evento histórico: la emisión, por parte de undeterminado hablante o escritor, de determinadas palabras (una oración) a una audiencia, con referencia a una situación, evento, o lo que sea, históricos" (J.L. Austin). Dicha competencia comunicativa nos viene presentada por Dell Hathaway Hymes, folclorista, antropólogo y por encima de todo sociolingüísta que nos dejó un gran legado pluricultural sobre la lógica pluridimensional que regula ya no solo el uso del lenguaje, sino la manera de ser y pensar en el mundo. Rescatando las palabras de Hymes (1964), los acontecimientos lingüísticos (speech events) no se explican por la sola aplicación de la competencia lingüística de los hablantes (lo que sería el código gramatical y sintáctico), porque la capacidad de hablar es inexplicable sin la de comunicar, que requiere el conocimiento implícito de normas psicológicas, culturales y sociales, éstas últimas están incluidas en el que llamamos código metarregulativo, del que también 
hacemos un uso inconsciente en toda acción comunicativa, o en todo "acto de habla"

\subsection{LA REFLEXIÓN CREADORA DEL LENGUAJE}

Necesitamos tres procesos, para empezar a entender cualquier acontecimiento lingüístico: el origen del signo, la lógica que regula el uso de ese signo en nuestras socioculturales, y el efecto del signo en el mundo. Sobre esta última acción, la gran respuesta nos viene dada de la mano de L. Wittgenstein, para quien: hablo me comunico por consiguiente existo en el mundo. En la evolución del pensamiento de Witthgenstein inclusive existe algo antes del lenguaje, como un silencio que no está detrás de las palabras sino antes.

Los expertos dividen la reflexión y aportes de L. Wittgenstein en dos momentos. Un "primer" Wittgenstein ${ }^{1}$ que hace una propuesta lógica filosófica para llegar a la esencia o forma misma del lenguaje privado que vaya más allá de la función declarativa de éste, puesto que el lenguaje ya no se concibe como figura de la realidad, sino como un instrumento, como una herramienta eidificadora de la realidad. Entender el mundo pasa por conocer las palabras en su uso cotidiano, ya que los juegos lingüísticos expresan la forma de la vida de un grupo cultural o comunidad interpretativa. En esos "juegos del lenguaje" barajamos la referencia y el sentido, pero en cuanto levantamos la mirada vemos el escenario completo, y de ahí que acto seguido pasemos a necesitar el "giro lingüístico" en el que quedan incluidos todos ellos.

Con nuestro lenguaje nos referimos a las cosas y con él decimos algo de ellas; llamamos a la primera de estas capacidades del lenguaje "referencia" y a la segunda sentido o significado. Presentamos a continuación un ejemplo de dicha multidimensionalidad del signo:

- Madame Roland, el día que fue condenada, se había vestido de blanco, muy cuidadosamente sus largos cabellos negros caían sueltos hasta su cintura, SENTIDO-A

- "De la boca de Madame Roland, una bonita mujer francesa salía el más puro y valiente lenguaje republicano", SENTIDO-B

Ambas expresiones comparten el mismo referente, se refieren a la misma persona, Madame Roland, pero expresan descripciones, detalles e informaciones distintas; tienen el mismo referente pero distinto sentido. Pero lingüísticamente hablando, Madame Roland, no es sólo una "representación isomórfica", sino que ésta en tanto que referencia tiene una vinculación con la realidad a la que sustituye, en palabras de Wittgenstein dentro de su teoría pictórica, sería una "relación figurativa" que es una consecuencia de la proyección.

Cuando relacionamos una figura con una cosa, objeto o ser, proyectamos dicha

\footnotetext{
${ }^{1}$ Su segunda propuesta filosófica está contenida en sus Investigaciones Filosóficas (1952) y esta época de su producción es llamada del "Segundo Wittgenstein". Ambas propuestas filosóficas hablan del lugar del lenguaje en la vida humana.
} 
figura en la realidad, dicho en otras palabras, la forma se gira para ver el escenario sociocultural en el que va a ser usado o va a tomar vida. La representación isomórfica se encarna en el mundo, y toma vida al ser acto de habla más allá de la mera forma: se inicia en vivo en un escenario sociocultural.

Dentro de esta denominación de "giro lingüístico", se agruparon a posteriori los aportes de diferentes autores y corrientes que coincidieron en destacar la importancia del lenguaje no ya solo en la construcción identitaria, sino afirmando que la performatividad del signo llegaba más allá: en la construcción de la realidad sociocultural. Cabe advertir que el giro lingüístico constituye el punto de partida desde el que emergen los distintos giros como el pragmático y el hermenéutico ${ }^{2}$, pero en modo alguno el giro lingüístico se corresponde con un paradigma o escuela teórica, ni constituye un solo movimiento contemporáneo ni es algo homogéneo. Bajo el apelativo de "giro lingüístico", confluye un nuevo orden interpretativo/reflexivo en los inicios del siglo XX sobre cómo funciona nuestra realidad, desde la relación sujeto/signo/comunidad interpretativo y que como esta tríada se encarna en nuestra cotidianidad. Una segunda advertencia, es sobre la no desaparición de la razón en el giro lingüístico, hablaríamos de una relegación y de una cambiante presencia en base a qué tipo de giro encarne en la realidad. Es decir, pese a que en un principio el "giro lingüístico" relega por completo el papel de la razón, ésta no desaparece sino que se desvanece como filtro cognoscitivo absoluto desde que en la Edad Moderna de Descartes, la razón erigiese como eje interpretativo con su dictum "Pienso luego existo". Lo que cambió en el siglo XX es justamente la importancia que se le otorgaba al lenguaje en el origen de nuestros actos, en primer lugar, y de cómo construimos nuestra convicción de lo que es real a partir de ese mismo lenguaje. En segundo lugar, tomando prestadas las tesis de Rafael Echeverría, al defender que este relevo interpretativo del lenguaje que desplaza a la razón, se inicia con Nietzsche, continúa con Wittgenstein, se consolida con Austin y luego después se completa con Searle.

Es desde esta óptica global y creadora del lenguaje, que el giro lingüístico aboga por abandonar el incierto mundo de los contenidos psicológicos, y pasa a centrarse en el análisis del lenguaje, para comprender al ser humano, al mundo, y al hombre en relación al mundo. Por otro lado, cabe evidenciar la conexión entre la base de la filosofía de Wittgenstein y la base filosófica del giro lingüístico. Pero además, podemos manifestar que el giro lingüístico bebe de la filosofía de Wittgenstein, en concreto desde la función descriptiva del lenguaje de este autor, función integrada en su Teoría pictórica o figurativa del significado.

Para entender lo que supuso el "giro lingüístico", como cambio reflexivo sobre el mundo, cabe hacer alusión a los discursos imperantes en la tradición filosófica respecto al lenguaje. Éste no era ni mucho menos obviado, puesto que la reflexión sobre el lenguaje existía, apero ésta se hacía desde su conceptualización de que el lenguaje humano era exclusivamente un mero medio

\footnotetext{
${ }^{2}$ El giro hermenéutico recoge los aportes de Von Humbolt (s. XIX) y Heidegger, más todos los ejes reflexivos del giro lingüístico, teniendo como principal contexto de plasmación, la filosofía alemana. Dentro de esta línea citaríamos a Gadamer, Ricoeur, Derrida y Vattimo.
} 
entre el sujeto y la realidad o el mundo, un mero medio exento por completo de la capacidad representativa, que es justo una de las inclusiones reflexivas claves para hablar del poder, la fuerza y la performatividad del lenguaje.

Antes del giro lingüístico, en la tradición filosófica, el lenguaje se valoraba sólo por su carácter asertivo ${ }^{3}$. Se defendía por consiguiente una comprensión del lenguaje declarativo sin integrar su valor representativo, sin llegar a reflejar el estado real de las cosas. Sólo a modo de muestra de esa puerta reflexiva sobre el lenguaje que abrieron Wittgenstein y el giro lingüístico, acabamos esta introducción teórica, con la conceptualización dada por $\mathrm{H}$. A. Murena sobre el arte como producto del intento de éste de regresar al origen del signo, que para este autor sería el silencio o "metáfora sagrada", puesto que nadie es autor de la voz, y conectaría con esa última idea de Wittgenstein sobre como antes de las palabras estaban los silencios. Murena expresa como lo que realmente mueve la producción artística no es ni provocar, interesar o transmitir sino intentar a reproducir su modelo originario, su matriz de multiplicación de las formas y de indeterminación del sentido. Derivado de esto, para Murena, "el arte, a través de la metáfora, viene a cambiar todos los lugares y criaturas del mundo, para que cada cosa viviente, al comprender que no es lo creía, pueda ser más, pueda ser cualquier otra cosa, todo lo que debe. El arte viene a salvar al mundo", a lo que añadimos, el arte viene a salvar al mundo desde la "metáfora sagrada". En este último reflexivo, entraríamos de pleno en el giro pragmático, cuya presentación se inicia en el siguiente epígrafe.

\section{DEL GIRO LINGÜÑISTICO AL GIRO PRAGMÁtICO TRASCENDENTAL}

Como primera proyectabilidad del lenguaje que integra el giro lingüístico, se pondera el lenguaje como un vehículo transparente para reflejar las representaciones del pensamiento, con una entidad propia que impone sus límites, (o como decía el propio L. Wittgenstein, "los límites de mi lenguaje son los límites de mi mundo") y determina en cierta manera, tanto al pensamiento como a la realidad. Desde esa capacidad representativa, los límites de mi mundo, son los límites de mi lenguaje, puesto que todos los objetos -reales y del pensamiento- son figurados por los signos (lingüísticos). En el giro lingüístico, desde los aportes de Wittgenstein, cuanto es decible puede, y debe, ser expresado con claridad, de lo contrario hay que callarlo, pues algo existe en cuanto es nombrado.

\footnotetext{
${ }^{3}$ Recordemos como Searle establece cinco tipos de actos de habla, en contraposición a los infinitos "juegos lingüístico" de Wittgenstein: asertivos o representativos, compromisivos o comisivos, directivos, declarativos y expresivos. Con esta categorización Searle, incide en que más allá de la correspondencia entre las palabas y el mundo (actos de habla asertivos o representativos), también la intencionalidad empodera a la palabra y ésta una vez empoderada toma una nueva dirección y protectabilidad de poder influir, remodelar, cambiar las formas sociales del mundo.
} 
Estas tesis de Wittgenstein, sientan las bases para entender la capacidad representativa que también defiende el giro lingüístico. Desde esta presentación general del "giro lingüístico", éste se materializa en diferentes vertientes y momentos de la reflexión filosófica y profana, y es entonces cuando empezamos a hablar de las diferentes corrientes del giro lingüístico, que a su vez, poseen heterogéneas nociones acerca de las característica y funciones del lenguaje, pero todas comparten esa visión de la capacidad representativa del lenguaje que hemos descrito.

En general se diferencian cuadro grandes líneas de reflexión derivados de dicho giro lingüístico: giro lingüístico analítico, giro pragmático, giro hermenéutico y giro pragmático-trascendental. Estos cuatro giros parten de dicha capacidad representativa, y por ende de desechar la concepción tradicional del lenguaje como un mero medio o instrumento para la designación de entidades independientes del lenguaje o para la comunicación de pensamiento prelingüisticos y en reconocer el papel constitutivo del lenguaje en nuestra relación con el mundo, pero cada uno de los giros, lo hará desde supuestos netamente diferentes.

\subsection{EL LENGUAJE EN LA CONSTRUCCIÓN DEL MUNDO VIDA}

Tal y como ya hemos enunciado de la mano de Searle, las palabras establecen puentes con el mundo, sus referentes, además los entes del mundo no se nos aparecen tomando como principal vía de conexión su cadena fónica, su grafía, sino los sentidos que se asocian a una imagen o representación mental. El verdadero poder y performatividad del lenguaje no es esa designación de un objeto por un nombre, sino en la intencionalidad de dicha designación y en la negociación o institucionalización de esta designación, ya que éstas últimas se insertan y producen en el plano cultural. Es impensable la perpetuidad diacrónica de dicha designación por una mera imposición o digamos una relación ostentiva directa. En primer orden, tendríamos que hablar de una relación indirecta en la cual los sentidos encarnados en el lenguaje, surgen de una intersubjetividad más un consenso colectivo institucionalizado, desde una perspectiva constructivista. El lenguaje es una Institución -tal y como diría Lévi Strauss-, una totalidad simbólicamente articulada, donde cada parte adquiere su significado por referencia al todo, es decir la cultura.

\subsection{EL DESCARNECIMIENTO DE LA RAZÓN EN EL LENGUAJE}

Más allá de la preeminencia del significado frente a la referencia, L. Wittgenstein imprime un carácter de verdad al lenguaje, desde el cuál se puede empezar a producir ese descarnecimiento de la razón en el lenguaje. Ese carácter de verdad empieza a entenderse en la propia función declarativa del lenguaje, pese a que no defienda que exista un orden perfecto del lenguaje, si reivindica como el lenguaje es el responsable del modo en que se nos aparecen los entes, pues sólo mediante el nombrar queda establecido lo que una entidad es, por lo cual lleva en sí la esencia del ser de los entes y la verdad de los mismos.

Esto es posible por esa superación de la mera racionalidad. No se trata de entender un objeto/ser/ característico, sino de crearlo y se crea cuando se 
produce a nivel inferencial y de forma automática la realidad figurativa de un objeto/ser o característica. El objeto/ser/ característica se crea por la simple producción a nivel inferencial de su representación. O lo que el propio Wittgenstein llama relación figurativa.

\section{RACIONALIDAD Y LENGUAJE}

La propuesta del giro pragmático trascendental intenta desde la proyectabilidad del lenguaje y la sostenibilidad de la razón, aunar esas dos vías reflexivas en apariencia opuestas para convertirlas en una única fuerza común reflexiva, tal y como nos señala Daniel Trapani -al introducir el pensamiento de Karl-Otto Apel, existen tradiciones filosóficas centradas en el lenguaje como el caso del giro pragmático que ya nacen con un nuevo espíritu creativo por el que "intentar ir con Kant más allá de Kant, con Heidegger más allá de Heidegger, con Vittgenstein más allá de Wittgenstein".

En este caso hacer desaparecer la razón de los distintos giros surge como de una errada estrategia de combatir los abusos de la razón desde la sin razón, cuando lo que se propone es una ampliación de la racionalidad que potencie las metas del lenguaje, optimizar la adecuación de los elementos constitutivos y de los sujetos de cualquier práctica comunicativa, preveer y mitigar la incertidumbre del conjunto social. De alguna manera que la racionalidad salga de su mera bidimensionalidad descriptiva y explicativa, para abrirse en su plena multidimensionalidad. En esta última junto a la racionalidad descriptiva y explicativa, pasaríamos desde el pensamiento de Karl-Otto Apel a hablar también de una racionalidad de la comprensión hermenéutica y de una racionalidad ética o ética discursiva desde la filosofía moral apeliana.

El giro herméneutico entre otros aportes se abre a una ética comunicativa, pues nos recuerda como las prácticas comunicativas más allá de poner en contacto a los seres humanos, a los ciudadanos, y de acortar distancias entre grupos socialmente distantes, lo que nos posibilita es dirigir nuestra acción social desde los valores. Hablamos de valores desde un consenso y coexistencia colectiva, pues pese a falsas interpretaciones el giro hermenéutico no nos habla del poder de las decisiones morales del sujeto, sino de la imposibilidad de separar hecho y valor en la acción social, lo que nos permitiría ir más allá de la mera reproducción de estructuras materiales 0 de un dictamen de la subjetividad. El giro hermenéutico nos traslada a la existencia del vivir como un hecho comunicativo de sentidos, conectando con el enfoque de Habermas de conducirnos hacia una esfera pública en la que vuelvan a visibilizarse los valores. A diferencia de los otros giros, en el hermenéutico la voz de la ética discursiva coexiste o como mínimo compite con los imperativos de la Tecnología: subjetividades que interactúan desde una ética discursiva.

Uno de los puntos de partida de la reflexión del giro hermenéutico, es la dinámica que ofrece para destruir los falsos límites del lenguaje, que se defendían en la tradición filosófica centrada en la Razón. La dinámica es rotunda, hace desaparecer el eje constitutivo del mundo vida desde la Razón. No olvidemos que inclusive en el giro pragmático, en el giro pragmático-trascendental y en el giro 
lingüístico analítico, la Razón está presente. Esta última manifestación se hace puesto que en los tres giros nombrados, la Razón aflora, aunque sea para deslegitimarla, o bien señalando las limitaciones impuestas a la Razón desde el lenguaje:

- En el giro lingüístico-analítico, las limitaciones impuestas a la Razón, provienen de esa "oculta estructura lógica y verdadera dellenguaje".

- Para el caso de los giros pragmático y pragmático-trascendental el límite de la Razón se centra en la "realización pragmática".

- El giro hermenéutico, reconoce el papel constitutivo del lenguaje en nuestra relación con el mundo, delegando por completo a la Razón, desde la constatación y revisión de la historia humana, desde la facticidad y contingencia de la pluralidad de lenguajes históricos, por la posibilidad de constituirse como auténticos seres sociales una vez adquirimos y dominamos el lenguajesimbólico.

Lo que nos dice el giro hermenéutico, es que no puedo comprender la abstracción constitutiva de mi mundo, hasta que no adquiero la inferencialidad de mi lenguaje simbólico.

La apertura al mundo se produce por la constitución de sentido que se da entre los hablantes tras el aprendizaje de una lengua (que precede a toda experiencia y teorización posible). La Razón en el giro pragmático-trascendental, está presente sin limitar al lenguaje sino al contrario, en su objetivo de reconstrucción racional de las condiciones que hacen posible llegar a un acuerdo intersubjetivo en la comunicación en el lenguaje ordinario y sostiene que en el lenguaje se encuentra el fundamento último de toda actividad racional, que el lenguaje no puede ser comprendido (vivenciado) con independencia del entendimiento (entendido éste como la Razón, como la parte de normativización y orden).

La Razón en el giro hermenéutico, también se rinde ante ese carácter recursivo del lenguaje (esta idea expresada del siguiente modo: "en el horizonte de sentido abierto por el lenguaje que sirve de límite a la razón"). Es a partir de relegar la Razón, que el mundo de vida se vuelve accesible y comprensible, y cuando el giro hermenéutico reconoce el papel constitutivo del lenguaje, en nuestra relación con el mundo, en la que la Razón, queda totalmente reconvertida.

\section{CONCLUSIONES}

Hemos visto como el lenguaje además de una dimensión sintáctica y semántica se proyecta también hacia una racionalidad de la comprensión hermenéutica y una ética discursiva. Desde el lenguaje las subjetividades hacen cultura, y defender una cultura comunicativa incluye reflexionar sobre la búsqueda de la resolución de los problemas desde el motor del orden moral. El lenguaje responde a una lógica de metarreglas y de valores.

Para huir de los desiertos reflexivos sobre nuestra realidad sociocultural, hemos de admitir que las palabras también están impregnadas de un sentido ético que pese a no constituir un orden universal, danzan en la creatividad humana 
cotidiana.

\section{BIBLIOGRAFÍA (APA)}

Apel, K.O. (1985). La transformación de la filosofía, 2 v. Madrid: Taurus.

Austin, J. (1981). Cómo hacer cosas con palabras. Barcelona: Paidós. Habermas, H. (1989). Teoría de la acción comunicativa. T. I. Madrid: Taurus. Hymes, D. (1964). Language in Culture and Society. New York \& London: Harper \& Row.

Searle, J. R. (1989). Actos de habla. Madrid: Cátedra.

Wittgenstein, L. (1961). Tractatus Logico-Philosphicus. Londres: Routledge. Investigaciones filosóficas. (1988). México: Crítica-UNAM. 